\title{
Collisional Energy Dependence of Peptide Ion Fragmentation
}

\author{
Pedatsur Neta, Yamil Simon-Manso, Xiaoyu Yang, and Stephen E. Stein \\ Chemical and Biochemical Reference Data Division, National Institute of Standards and Technology, \\ Gaithersburg, Maryland, USA
}

\begin{abstract}
The energy dependence of fragmentation in a collision cell was measured for 2400 protonated peptide ions derived from the digestion of 24 proteins. The collision voltage at which the sum of the fragment ion abundances was equal to the remaining parent ion $\left(V_{1 / 2}\right)$ was the principal measure of fragmentation effectiveness. Each class of peptides was characterized by a linear relation between $V_{1 / 2}$ and $\mathrm{m} / z$ whose slope depended on the peptide class and, with little adjustment, intersected the origin. Peptide ions where the number of protons is no greater than the number of arginine residues show the greatest slope, $V_{1 / 2} /(\mathrm{m} / \mathrm{z})=0.0472$ (all slopes in units of $\mathrm{V} \mathrm{Da}^{-1} \mathrm{e}$ ). For peptides where the number of protons is greater than the number of arginines, but not greater than the total number of basic residues, the slope decreases to 0.0414 for singly charged ions, 0.0382 for doubly charged, 0.0346 for triply charged, and 0.0308 for more highly charged ions. With one mobile proton, the slope is about 0.029 for singly and doubly charged ions and slightly lower for more highly charged ions. With two or more mobile protons the slope is 0.0207 . By removing $m / z$ dependence, the deviation of $V_{1 / 2}$ from a line provides a relative measure of the ease of fragmentation of an ion in each class. This information can guide the selection of optimal conditions for tandem mass spectrometry studies in collision cells for selected peptide ions as well as aid in comparing the reactivity of ions differing in $m / z$ and charge state. (J Am Soc Mass Spectrom 2009, 20, 469-476) (C) 2009 Published by Elsevier Inc. on behalf of American Society for Mass Spectrometry
\end{abstract}

$\mathrm{T}$ The principal source of variation in the collision cell tandem mass spectrum (MS/MS spectrum) of a given ion is collision energy. With increasing collision energy, initially formed product ions possess increasing amounts of translational energy and thus undergo increasing degrees of fragmentation in subsequent collisions. This behavior is ion structure specific and not readily modeled, although its general features are well known. The main features are that fragmentation requires more energy with increasing peptide ion mass, decreasing charge, and increasing number of basic amino acid residues [1, 2]. Furthermore, acetylation of the $\mathrm{N}$-terminus or side-chain amino groups facilitates fragmentation by decreasing basicity. Quantitative relationships between peptide ion mass and efficiency of collisional fragmentation has been derived for doubly charged peptides [3]. More recently, effects of specific amino acid residues on the relative facility of specific peptide bond fragmentations have been analyzed in detail $[4,5]$.

Although various molecular factors that influence fragmentation rates are known, their influence on relative extents of fragmentation in collision cells has not been measured. In particular, it is difficult to compare the inherent resistance to dissociation of ions with different $m / z$ or charge states. In this work we do this by

Address reprint requests to Dr. Steve Stein, NIST Mass Spectral Data Center, A260/222, Gaithersburg, MD 20899. E-mail: steve.stein@nist.gov examining the influence of $\mathrm{m} / \mathrm{z}$ on the energy needed to achieve a given extent of dissociation for different classes of peptide ions. The data were acquired as part of our reference data measurement program, where it has been our practice to measure reference spectra over a wide range of collision energy, typically at 20 intervals, where dissociation ranges from little to complete dissociation of the parent ion. From this information it is straightforward to derive the collision energy for each ion at which it undergoes a given extent of dissociation. Results of this analysis are presented in this work at energies where detected amounts of product ions are equal to the remaining parent, which should correspond roughly to a $50 \%$ extent of dissociation.

\section{Experimental $^{\mathbf{1}}$}

\section{Materials}

The following proteins were used: bovine: serum albumin (BSA), actin, carbonic anhydrase $2, \alpha$-caseine, $\beta$-caseine, $\alpha$-lactalbumin, $\beta$-lactoglobulin, cytochrome $\mathrm{C}$, transferrin, and ubiquitin; rabbit: glyceraldehyde-3-phosphate dehydrogenase and phosphorylase-b; chicken: ovalbu-

\footnotetext{
${ }^{1}$ Certain commercial equipment, instruments, or materials are identified in this document. Such identification does not imply recommendation or endorsement by the National Institute of Standards and Technology, nor does it imply that the products identified are necessarily the best available for the purpose.
}

(C) 2009 Published by Elsevier Inc. on behalf of American Society for Mass Spectrometry. 1044-0305/09/\$32.00

doi:10.1016/j.jasms.2008.11.005
Published online November 21, 2008 Received May 7, 2008 Revised November 3, 2008 Accepted November 6, 2008 
min and lysozyme; horse heart myoglobin; E. coli: $\beta$-galactosidase and mannose-6-phosphate isomerase; human: $\alpha_{1}$ acid glycoprotein, fibrinogen, hemoglobin, superoxide dismutase, and transferrin; and horseradish peroxidase. These proteins, $\alpha$-chymotrypsin from bovine pancreas, DL-dithiothreitol (DTT), and iodoacetamide were obtained from Sigma (St. Louis, MO, USA) in their highest purity available. Sequencing-grade modified trypsin was obtained from Promega (Madison, WI, USA). Tris(hydroxymethyl)aminomethane (Tris buffer; ultrapure, 99+\%) was from Aldrich Chemical (Milwaukee, WI, USA). Urea (>99.5\%), ammonium hydrogen carbonate ( $>99.5 \%$ ), and formic acid (puriss., $50 \%$ in water) were from Fluka (St. Louis, MO, USA). Acetonitrile was high-performance liquid chromatography (HPLC) grade from Spectrum and water was purified with a WaterPro PS system (Labconco Corp., Kansas City, MO, USA).

\section{Methods}

In some experiments BSA was digested by trypsin directly (without reduction and alkylation). BSA (1 mg) was dissolved in $1 \mathrm{~mL} \mathrm{NH}_{4} \mathrm{HCO}_{3}\left(50 \mathrm{mmol} \mathrm{L}^{-1}\right.$, $\mathrm{pH} 7.7$ to 8.0 ) and digested with $20 \mu \mathrm{g}$ trypsin at $37^{\circ} \mathrm{C}$ for $16 \mathrm{~h}$. Proteins that do not contain cysteine were also digested by this method. Most proteins were reduced and alkylated before digestion. Reduction with DTT and alkylation with iodoacetamide were carried out in $6 \mathrm{~mol} \mathrm{~L}^{-1}$ urea/0.1 mol L $\mathrm{L}^{-1}$ Tris buffer solutions at room temperature, followed by $10 \times$ dilution with water and digestion with trypsin at $37^{\circ} \mathrm{C}$, as previously described [6]. A similar procedure was used for the digestion of alkylated BSA by chymotrypsin. After digestion, the solutions were acidified with about $10 \mu \mathrm{L}$ of $50 \%$ formic acid and refrigerated until use.

The first experiments were carried out with BSA, using trypsin and chymotrypsin to obtain different sets of peptides. The 23 other proteins were digested with trypsin only. The fragmentation patterns of the protonated peptide ions were acquired with a triple quadrupole mass spectrometer with electrospray ionization (ESI). The triple quadrupole offered a well-defined and adjustable collision voltage and independent adjustment of collision cell pressure. However, direct liquid chromatography-tandem mass spectrometry (LC-MS/ MS) measurements in such an instrument could not provide the signal strengths sought in this work. Therefore, we chose to fractionate the LC eluent into 80 to 90 fractions and to analyze each fraction independently.

Protein digest solutions were analyzed by HPLC using a Waters 2695 Separation Module with a Waters 2996 Photodiode Array detector (Waters Corp., Milford, MA, USA). The column was a Waters Atlantis $\mathrm{dC}_{18} 3$ $\mu \mathrm{m}(2.1 \mathrm{~mm} \times 50 \mathrm{~mm})$, held at $30^{\circ} \mathrm{C}$, and the eluents were water and acetonitrile, each containing $0.1 \%$ formic acid. The eluent consisted of $99 \%$ water for the first $2 \mathrm{~min}$, then a gradient was applied to change the water content from 99 to $67 \%$ in $80 \mathrm{~min}$ (all the peptides eluted within this time), after which the water content was decreased to $20 \%$ within $5 \mathrm{~min}$ and held there for an additional $2 \mathrm{~min}$ to elute undigested protein and large fractions, and finally the column was equilibrated for 10 min in the original eluent composition of $99 \%$ water. The flow rate was $0.2 \mathrm{~mL} / \mathrm{min}$ throughout. After establishing the conditions for the above-cited experiments, the protein digests were separated by HPLC by collecting 80 to 90 fractions ( 1 min each) from each of 15 to 20 consecutive runs to obtain sufficient amounts of solution for subsequent mass spectrometric measurements. During this process, the original digest was held at $4{ }^{\circ} \mathrm{C}$ and the collected fractions were held at $5-7^{\circ} \mathrm{C}$.

The collected fractions were analyzed by ESI mass spectrometry using a Micromass Quattro Micro API Benchtop Mass Spectrometer (Waters Corp.). The solution was injected with a syringe at a flow rate of 10 $\mu \mathrm{L} / \mathrm{min}$ and the positive-ion spectrum was recorded in a "continuum" mode from $\mathrm{m} / \mathrm{z} 100$ to $\mathrm{m} / \mathrm{z} 1900$ at different cone voltages (in most cases between 15 and 30 $\mathrm{V})$. The main peaks were then selected for MS/MS measurements at their respective optimal cone voltage (in some cases $>30 \mathrm{~V}$ ). Each precursor ion peak was examined at 20 different collision voltages and the fragment peaks were measured in a "centroid" mode, generally between $\mathrm{m} / \mathrm{z} 40$ and $\mathrm{m} / \mathrm{z}$ 1900. The MS/MS runs, lasting between 15 and $50 \mathrm{~min}$, depending on the peak intensities, were cycled repeatedly through the 20 collision voltages to enhance the reproducibility of the relative peak intensities at the different voltages. Argon was used in the collision cell, at a pressure of $0.21 \mathrm{~Pa}$ (1.6 mTorr). In several cases, the MS/MS spectra were also measured at pressures of $0.4,0.8$, and 2.8 mTorr. The rate of fragmentation was lower at lower Ar pressure $\left(V_{1 / 2}\right.$ increased by about $60 \%$ at $0.8 \mathrm{mT}$ Torr and another roughly $80 \%$ at 0.4 mTorr) but remained essentially the same when the pressure was increased from 1.6 to 2.8 mTorr.

\section{Data Processing}

MS/MS spectra were acquired in "centroid" mode, whereby signals within each individual time interval in a given spectrum were centered and integrated by the instrument data system. Spectra were produced with an average spacing of $0.8 \mathrm{~m} / \mathrm{z}$ between adjacent peaks throughout the $m / z$ range. Occasional noise spikes were also produced, typically generating a peak more than 10-fold greater than the typical background signal several times per spectrum. For each precursor ion, spectra for 20 different collision energies were acquired using acquisition software to step through a selected collision voltage range, generating a single spectrum for each collision voltage for a single cycle. Depending on the length of acquisition time and maximum $m / z$, the number of such spectra at each collision voltage varied from 13 to 130, longer times being used for weaker signals. To find peaks, first a summary spectrum was created by summing the abundance of each peak in vector ele- 
ments of $0.1 \mathrm{~m} / \mathrm{z}$ width. These bins were then sorted by abundance and the $\mathrm{m} / \mathrm{z}$ values of the largest elements were examined in order of decreasing abundance. For each element, all abundances within $0.3 \mathrm{~m} / \mathrm{z}$ (3 bins higher and lower) were added, although peak abundances included in an earlier peak were not reused. Some spikes, generally present as 10 - to 20 -fold the background signal level, typically occurred several times per spectrum. For each maximum, exact $\mathrm{m} / \mathrm{z}$ values were computed by weighting individual peak abundances and exact $\mathrm{m} / \mathrm{z}$ values. The median abundance of all peaks was used as a measure of random noise. Its value was found to be independent of $\mathrm{m} / \mathrm{z}$ value and collision voltage and had a typical value of 200 abundance units. Tentatively identified peaks with abundances less than 5-fold the noise level were discarded. A further selection criterion was used that required peaks present at less than 6-fold the noise level to be present in the spectrum for next higher or lower collision voltage. Final abundances are reported as signal to noise units multiplied by 100 , obtained after subtracting the median noise signal from each measured peak.

\section{Results and Discussion}

The first, and most detailed, experiments were carried out with BSA, digested by trypsin. The peptides formed were separated by liquid chromatography and collected in 80 fractions. The MS and MS/MS spectra of these fractions permitted identification of 105 peptides, many of which were observed as positive ions in different charge states. The intensities of the MS peaks in the eluent varied by several orders of magnitude. All the large peaks were analyzed by MS/MS. Ion peaks that were as low as $1 \%$ of the highest peak in a spectrum could often be analyzed and assigned successfully (if the peptide was relatively small). Peaks that were much less than $1 \%$ were also observed, although attempts to analyze them by MS/MS to determine their amino acid sequence generally failed because of very weak fragment peaks.

The amino acid sequence of the peptide ions was manually determined from the MS/MS spectra with the aid of Protein Prospector (http:/ / prospector.ucsf.edu). The peptides identified in BSA digests represent complete coverage of the BSA amino acid sequence. Many of the peptides are those expected from enzymatic cleavage at the C-terminus of all lysine and arginine residues, except when proline was on the C-terminus. These peptides cover $83 \%$ of the amino acids in BSA. Other peptides contain one or more missed cleavages and they cover the rest of the amino acids, sometimes overlapping regions of other observed peptides (with missed cleavages at different sites). All the missed cleavages are at sites containing an acidic amino acid, asparate or glutamate, next to the lysine or arginine, on either side [7]. However, some sites of this type did undergo tryptic cleavage. Peptides with N-terminal glutamine were also observed in their deaminated form (i.e., after conversion of glutamine to pyroglutamate). One peptide containing S-carbamidomethylcysteine at the N-terminus also underwent loss of $\mathrm{NH}_{3}$ to form a cyclic product [8]. Peptides containing methionine or tryptophan were observed in the original form and frequently also in an oxidized form.

Trypsin cleaves proteins predominantly at the Cterminus of lysine and arginine, thus providing at least one basic amino acid in each peptide. In contrast, chymotrypsin cleaves mainly at the C-terminus of aromatic amino acids and leucine, thus producing a whole set of different peptides, some of which did not contain any basic amino acid residue. In all, 81 peptides were identified following chymotryptic digestion of BSA, many of which were observed in several charge states. These peptides provide slightly less than complete coverage of the BSA amino acid sequence. It should be noted that in the analysis in the following text we find no distinction between tryptic and chymotryptic peptides; i.e., whether the basic residue is located at the terminus or elsewhere does not affect the rate of fragmentation.

The other proteins were digested by trypsin only. Altogether, nearly 2500 MS/MS spectra were recorded and most of them led to positive identification of the peptide ion. The present collection of spectra represents the distribution of peptides from 24 proteins and, as such, differs from other collections that have been used to examine variations among peptide spectra. Other collections were built from spectra identified by library search algorithms and thus contain only spectra that possessed sufficiently informative fragmentation patterns. In the present work, all major peaks originating from the digests have been identified, so relatively uninformative spectra are included. Further, the use of a triple quadrupole in the present work allows collection of low mass (primarily immonium) ions and permits straightforward measurement of collisional energy, neither of which may be done in ion trap instruments.

\section{Fragmentation versus Collision Voltage}

Tandem mass spectra were acquired as a function of collision voltage for all peptides. Signal strength, scattering, and peak broadening at high collision voltage limited this range somewhat, although spectra for almost all detected peptide ions could be acquired at conditions where dissociation of the parent ion was nearly complete. A measure of the propensity of a given peptide ion to dissociate was obtained by finding the collision voltage necessary for $50 \%$ fragmentation, $V_{1 / 2}$, using linear interpolation of the fixed collision voltage measurements. $V_{1 / 2}$ is defined as the energy at which the sum of product ion abundances equals the remaining precursor ion abundance. In calculating $V_{1 / 2}$ we considered how to take into account the ions formed by neutral loss of water or ammonia from the precursor 
ions, which are important in certain cases. Since the magnitude of these loss peaks could vary greatly between ions, and backbone fragmentation is of more interest, the abundance of such peaks was added to the abundance of the remaining parent ion when computing $V_{1 / 2}$. This, however, had very little effect on the computed slopes.

A plot of $V_{1 / 2}$ versus $m / z$ for all peptide ions shows a wide scatter of data points pointing in the direction of the origin but varying in slope by a factor of $>2$ (Figure 1; data points bracketed by two lines with the extreme slopes are discussed in the following text). Linear correlation of all the data in Figure 1 gives a slope of 0.031 but with a correlation coefficient of only 0.68 . Careful examination of the data showed that different categories of peptide ions fell on lines having far less dispersion and higher correlation coefficients. Therefore, each group was plotted separately. First, we separated the peptide ions into those with mobile protons (number of charges greater than the sum of $\mathrm{R}, \mathrm{K}$, and $\mathrm{H}$ residues) and those with no mobile protons (number of charges equal to or less than the sum of $\mathrm{R}, \mathrm{K}$, and $\mathrm{H}$ residues). Although the first group showed some dependence on total number of charges, the second group showed strong dependence and was subgrouped by charge. Furthermore, if the peptide contained no more charges than arginines, its fragmentation required more energy than a peptide containing $\mathrm{K}$ or $\mathrm{H}$, which are known to have lower basicity.

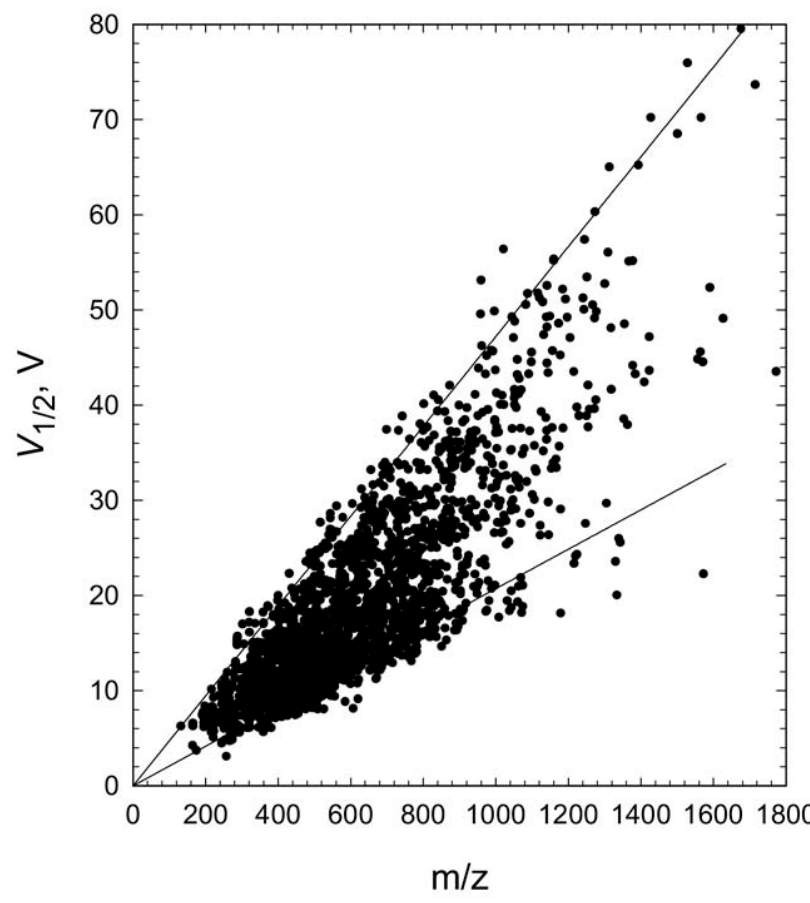

Figure 1. Correlation between the voltage for $50 \%$ fragmentation $\left(V_{1 / 2}\right)$ and the $m / z$ values for all peptide ions examined. The data points are bracketed by two lines with the extreme slopes discussed in the text below.
Figure 2a shows a plot for peptide ions with the number of protons no greater than the number of $R$ residues (one or two). The line represents the correlation $V_{1 / 2}=(0.0472 \pm 0.0003)(\mathrm{m} / \mathrm{z})$ with a correlation coefficient $R^{2}=0.974$. The slope is given in units of $\mathrm{V}$ $\mathrm{Da}^{-1}$ e throughout this report and its associated uncertainty is given as one standard deviation. If the line is not forced to go through the origin, a small intercept of $(1.0 \pm 0.5) \mathrm{V}$ is calculated with a similar slope $(0.0460 \pm$ $0.0007)$ and identical $R^{2}$. In this group of peptide ions, 4 ions (SDPR, DPER, LTDDPR, and IDPNAWVER, open circles in Figure 2a) were found to have $V_{1 / 2}$ values lower by $19-51 \%$ from the calculated line. These peptides contain the amino acid sequence DP, which appears to facilitate fragmentation because of a combination of the known effects of proline and aspartic acid, and were not included in the correlation. It should be noted that 29 other peptides in this group contain D not adjacent to P. Of these, 20 had $V_{1 / 2}$ values lower than those calculated from the slope by an average of $5.5 \%$ and 9 had $V_{1 / 2}$ values higher by an average of $3.8 \%$. Thus, although the presence of D in R-containing peptides with no mobile protons is known to give dominant peaks as a result of fragmentation next to $D[9,10]$, this effect is not accompanied by an enhanced rate of peptide fragmentation (this effect is under further study in our lab).

Peptide ions containing $\mathrm{K}$ or $\mathrm{H}$, with or without $\mathrm{R}$ and no mobile protons, were subgrouped by their total charge. For charge +1 (Figure $2 b$ ) the slope is $(0.0414 \pm 0.0002)\left(R^{2}=0.963\right)$; for charge +2 (Figure $2 \mathrm{c})$ the slope is $(0.0382 \pm 0.0002)\left(R^{2}=0.952\right)$; and for charge +3 (Figure $2 \mathrm{~d}$ ) the slope is $(0.0346 \pm 0.0004)$ $\left(R^{2}=0.888\right)$. In Figure $2 \mathrm{~d}$ the data show greater deviations from the calculated line, compared with the previous figures. This appears to be the result of the distribution of basic residues within the peptide. When three basic residues are close together and thus the protons are concentrated on one part of the peptide chain, the neutral residues at the other end of the peptide are readily lost as neutral fragments. This is exemplified by the peptides SRRHPEYAVSVL, LLGNVLVCVLAHHFGK, and DIVNMLMHHDR. With charge +4 the data were also scattered, probably for the same reason. The plot (not shown) gives a lower slope, $(0.0308 \pm 0.0005)$, and a lower correlation coefficient $\left(R^{2}=0.802\right)$.

A large number of peptide ions with one mobile proton are found in the present collection. A plot of all the data points (Figure 3 ) gives a linear correlation but with a wide scatter $( \pm 25 \%)$ in the value of $V_{1 / 2}$ at the same $m / z$ value. The calculated slope from all the points is 0.0277 , with $R^{2}=0.878$. An attempt to distinguish between peptides containing only $R$ and those containing $\mathrm{K}$ or $\mathrm{H}$ showed no clear distinction, in contrast with the case of peptide ions with no mobile protons discussed earlier. An attempt to separate the peptides into subgroups according to the total charge showed some distinction, as seen in the different color points in Figure 3. Although there is much overlap among the 


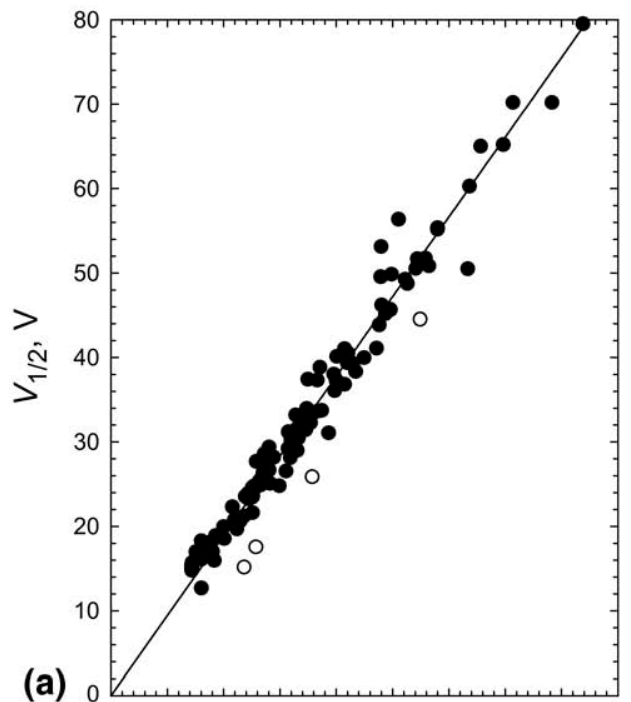

(b)
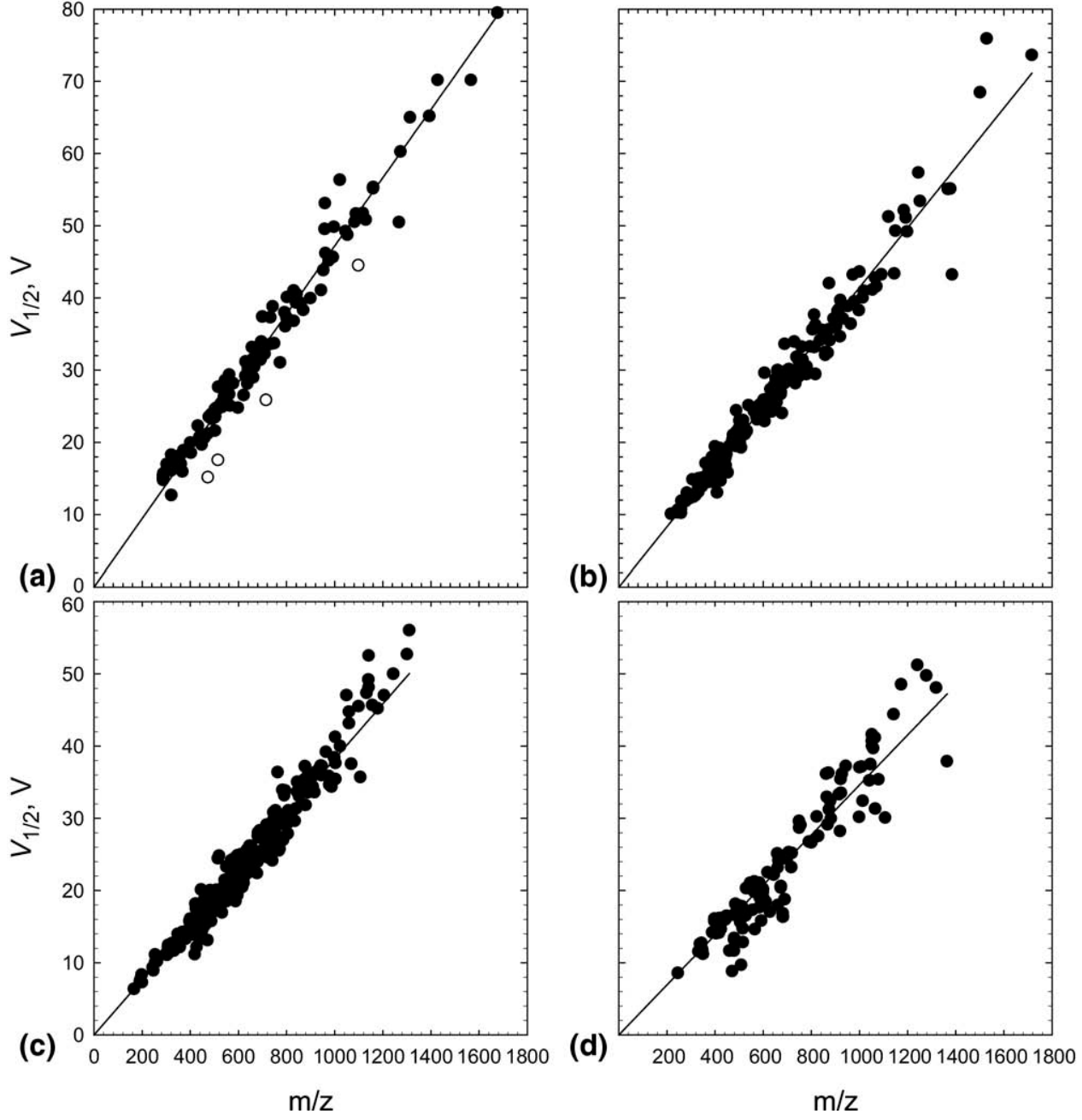

Figure 2. Correlation between $V_{1 / 2}$ and $\mathrm{m} / \mathrm{z}$ for peptide ions with no mobile protons. (a) Peptide ions containing one or two arginines and the same number of protons; other basic residues ( $\mathrm{K}$ or $\mathrm{H}$ ) may be present but with no corresponding protons. (b) Singly protonated peptide ions with no mobile protons and with $\mathrm{K}$ or $\mathrm{H}$ as the basic residues. (c) Doubly protonated peptide ions with no mobile protons and with at least one of the basic residues being K or H. (d) Triply protonated peptide ions with no mobile protons and with at least one of the basic residues being $\mathrm{K}$ or $\mathrm{H}$.

different groups in this plot, we derived correlations for each group separately to outline possible differences. Peptide ions with one mobile proton and total charge +1 (no basic residues) fit a slope of $(0.0286 \pm 0.0006)$ with $R^{2}=0.883$; those with charge +2 are the most abundant and show a slope of $(0.0294 \pm 0.0001)$ with $R^{2}=0.926$; those with charge +3 fit a slope of $(0.0263 \pm$ $0.0002)$ with $R^{2}=0.872$; those with charge +4 fit a slope of $(0.0242 \pm 0.0003)$ with $R^{2}=0.851$; and about 40 peptide ions with a charge between +5 and +7 fit a slope of 0.0237 but with $R^{2}$ only 0.788 . The slope calculated from all the points together $(0.0277)$ is near the average of the values for the doubly and triply charged ions, the most abundant in this group. Singly charged are the least abundant and thus their slope has the highest uncertainty. Thus, grouping by charge shows a minimal difference between singly and doubly charged ions but clearer differences between these and the more highly charged ions.
A number of peptide ions with two or more mobile protons were also found in this collection. These ions show the lowest slope, $(0.0207 \pm 0.0001)$, with $R^{2}=$ 0.835 (Figure 4). Clearly, the slope of the correlation line increases as the binding of the least tightly bound proton increases. The increase in ease of dissociation with increasing charge is perhaps connected to coulombic relief and differences in the stabilities of the unheated ion, although the significant sensitivity differences in the mobile and no mobile proton classes is unexplained.

Peptides lacking a free terminal $\mathrm{NH}_{2}$ group can be included in the correlation, but with some modification. Acetylation of the amino group or conversion of glutamine to pyroglutamic acid lower the basicity of the $\mathrm{N}$-terminus and thus may facilitate fragmentation. This effect, however, should depend on the availability of protons. We examined 9 such peptide ions with no mobile protons and 9 with one mobile proton. In the 


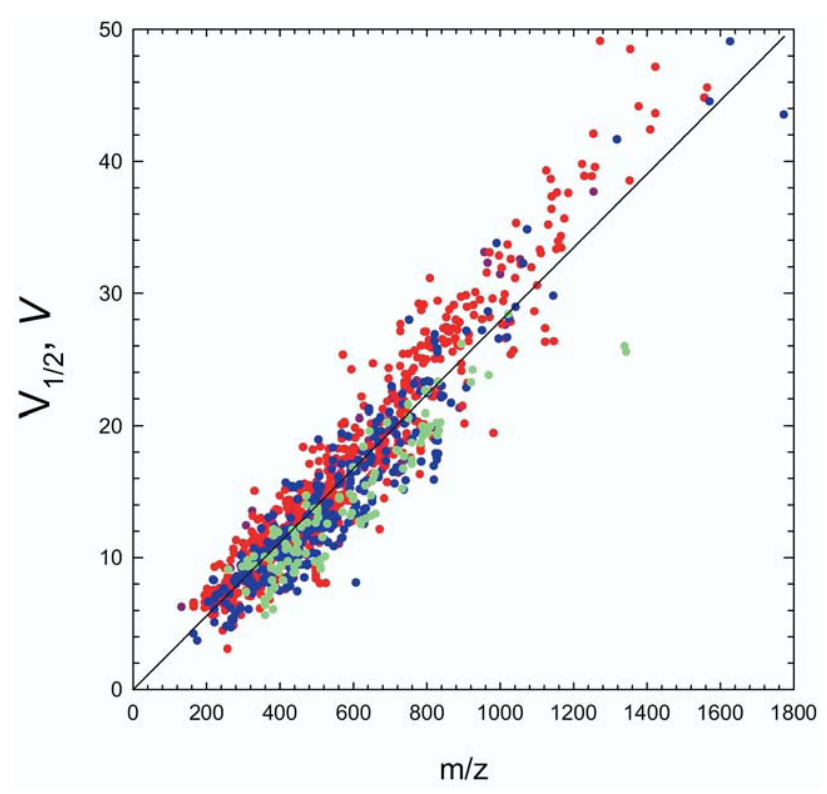

Figure 3. Dependence of $V_{1 / 2}$ on $\mathrm{m} / \mathrm{z}$ for peptide ions with one mobile proton. Purple, $1+$; red, $2+$; blue, $3+$; green, $4+$.

absence of mobile protons the values of $V_{1 / 2}$ differed little from the values expected for the unmodified $\mathrm{N}$-terminus (average deviation of $3 \%$ ). On the other hand, peptide ions bearing one mobile proton exhibited an average decrease in $V_{1 / 2}$ of $29 \%$ upon such terminal amino group modifications. In fact, these $V_{1 / 2}$ values for such peptide ions were very close to the calculated values if they were assumed to have two, instead of one, mobile protons. Thus it is possible to include such peptides in the general correlations discussed earlier if they contain no mobile protons and, if they contain one mobile proton, by assigning them an additional mobile proton.

In the subgroup of peptide ions with one mobile proton and a charge of +2 there are over 550 data points. The extreme spread in the values of $V_{1 / 2}$ is $\pm 7 \mathrm{~V}$ from the calculated line. However, $90 \%$ of the points

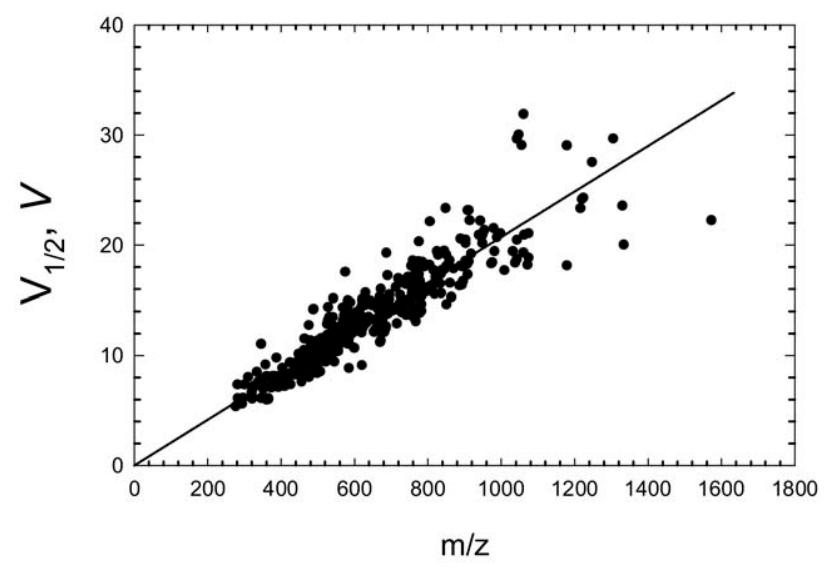

Figure 4. Correlation between $V_{1 / 2}$ and $m / z$ for peptide ions with two or more mobile protons.

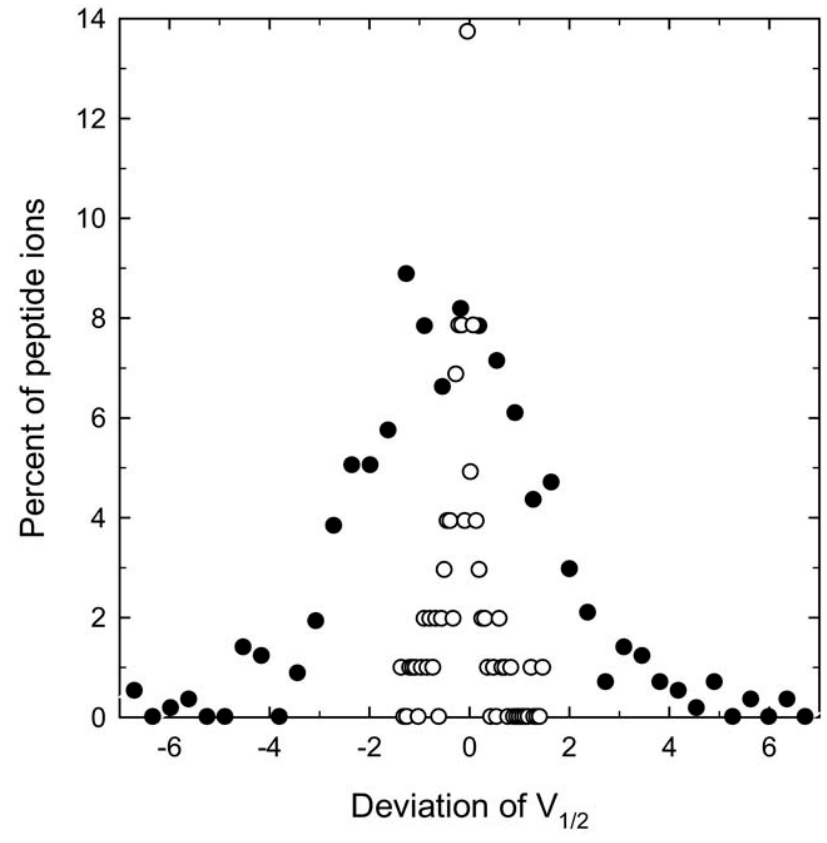

Figure 5. Percentage of peptide ions, doubly charged with one mobile proton, showing various degrees of deviations from the correlation of $V_{1 / 2}$ with $\mathrm{m} / \mathrm{z}$ (solid circles) and a similar distribution for some peptide ions that were each measured twice (open circles).

are within $\pm 3 \mathrm{~V}, 75 \%$ are within $\pm 2 \mathrm{~V}$, and $50 \%$ of the points are within $\pm 1.2 \mathrm{~V}$. For peptide ions that were measured twice, $90 \%$ of the data are reproducible within $\pm 0.8 \mathrm{~V}$, indicating that the deviations in the present set of data are almost 4 -fold greater than the experimental uncertainties (see Figure 5). These deviations are clearly attributed to the wide variety of peptide structures, which may influence the fragmentation rate. Examination of the peptide ions with the largest positive deviation revealed that many of them contain $P$ next to the N-terminus (e.g., YPVEPFTER). Since doubly charged peptide ions commonly fragment to produce major $b_{2}$ and $\mathrm{y}_{n-2}$ peaks and since $\mathrm{P}$ promotes fragmentation at its N-terminus but inhibits fragmentation at its Cterminus, it was speculated that this specific location of $\mathrm{P}$ is the cause for the higher $V_{1 / 2}$ value.

To examine the variations of $V_{1 / 2}$ values at fixed $\mathrm{m} / \mathrm{z}$, modifications to the sequence of a specific outlier doubly charged peptide ion, YPVEPFTER, were made. For this peptide $V_{1 / 2}$ was found to be $22 \%$ higher than the value calculated from the derived slope. Interchanging the PV sequence to form the peptide YVPEPFTER decreased the $V_{1 / 2}$ value by $36 \%$. The result for this peptide becomes $22 \%$ lower than calculated-i.e., $\mathrm{P}$ in the third position facilitates fragmentation at this highly reactive bond (the resulting $\mathrm{y}_{7}$ peak was more than twice as large). Interchanging other amino acids led to variations of roughly $10 \%$.

For comparison, among the doubly charged subgroup, 27 peptide ions were found with $\mathrm{P}$ in the third position. Of these, 21 show $V_{1 / 2}$ values lower than 
calculated, by an average of $20 \%$ but with very wide variations, and 6 show higher values. Among all the doubly charged ions with one mobile proton are found 150 peptide ions that contain $\mathrm{P}$ at positions other than the second and third from the N-terminus. Among these ions, 82 have $V_{1 / 2}$ values lower than calculated from the derived slope by an average of $13 \%$ and 68 have $V_{1 / 2}$ values higher than calculated by an average of $9.6 \%$, with wide variations and no clear trends. These comparisons suggest that, in this type of peptide ions, $\mathrm{P}$ at position 2 inhibits fragmentation, at position 3 facilitates fragmentation, whereas at other positions has generally minimal effects.

Doubly charged peptide ions with one mobile proton showed the most dispersion. If one were to use the correlation for these ions to predict the collision voltage needed for $50 \%$ dissociation, then for half the peptides the percentage dissociation would vary between 40 and $60 \%$ for peptide ions with $\mathrm{m} / \mathrm{z}$ near 1000 but between 30 and $80 \%$ for peptide ions with $\mathrm{m} / \mathrm{z}$ near 250 . For some peptide ions, however, the deviations will be larger and, in the most extreme cases, the extent of dissociation may vary between 10 and $95 \%$. These margins are similar or slightly tighter for the other groups of peptides. Thus for half the peptide ions it is possible to calculate the collision cell voltage setting required to give a reasonably detailed MS/MS spectrum simply by categorizing the peptide ion into one of the groups discussed earlier and using the slope derived for that group. It should be stressed that this derivation holds for peptides with terminal nonderivatized $\mathrm{NH}_{2}$ group, that peptides containing only arginine and no mobile protons show considerably lower $V_{1 / 2}$ values if they contain the sequence DP but not D or P alone, and that in highly charged ions the concentration of charges along the peptide chain has an effect on the fragmentation rate. The presence of $\mathrm{P}$ in peptides with mobile protons may lead to preferential fragmentation at the $\mathrm{N}$-terminal of $\mathrm{P}$ but appears to have a generally minimal effect on the value of $V_{1 / 2}$, except when $\mathrm{P}$ is located at position 2 or position 3 from the $\mathrm{N}$-terminus as discussed earlier. Similarly, the presence of acidic groups has no pronounced effect on $V_{1 / 2}$.

Our results reinforce and extend the observations made by Haller et al. [3] for doubly charged tryptic peptides, that dissociation rates depend linearly on the precursor mass. The present data also show that this relation holds regardless of charge state. These trends are qualitatively consistent with the idea that the dissociation rate of a peptide ion depends on its energy per oscillator. That is, to achieve a specific dissociation rate, an increase in the number of atoms in a peptide must be compensated by a corresponding increase in vibrational energy. An increase in vibrational energy can be delivered by increasing collision voltage or by higher charge state. To illustrate, if two ions had the same $m / z$ but differed in charge by a factor of two, the higher charge state ion would have twice the mass and therefore require twice the amount of energy (heat capacity) than the lower charge state ion to reach the same level of excitation. However, the higher charge state ion would also have twice the collisional energy at a fixed collisional voltage. Thus these two ions should each acquire similar amounts of energy per oscillator at a given voltage and thereby achieve a similar degree of excitation.

The linearity of the plots suggests that the energy deposited into the peptide ions is linearly related to the collision voltage. Since in collision cells operating in the mTorr region, there are sufficient collisions to translationally cool the ion, the possibility that a fixed fraction of the collisional energy is converted to internal energyregardless of the specific collision cross section-does not seem unreasonable. The different slopes for different charge states of peptides with no mobile protons may be attributable to the different stabilities of the ions; folded structures expected for singly charged ions might require additional energy (higher "heat capacity") to unfold at higher energies and coulombic repulsion may contribute to rates of higher charge state dissociation. Charge effects for ions with mobile protons, however, do not follow such trends. Singly charged ions in this class are unique in their lack of basic residues: the possibly more variable location of the mobile protons may add to the dispersion of $V_{1 / 2}$ values. Studies are under way in our lab to clarify these effects.

\section{Selecting Collision Energies}

The present results can be used to assess the degree of variation in extent of dissociation in practical experiments where peptide identity and/or charge state are not known or not used for selecting collision energy. If only precursor $\mathrm{m} / \mathrm{z}$ is used in selecting collision energy, and a $50 \%$ dissociation target is desired, one would set this energy to $0.0307 \mathrm{~m} / \mathrm{z}$ (slope in Figure 1). In this case $53 \%$ of peptide ions in this collection dissociate between 10 and $90 \%$, but $21 \%$ of the ions dissociate $<10 \%$ and $26 \%$ of the ions dissociate $>90 \%$. Not surprisingly, the $21 \%$ showing little dissociation predominantly (99\%) are those with no mobile protons and the $26 \%$ showing very high dissociation have one or two mobile protons. Although the number of mobile protons cannot be determined a priori, high-resolution instruments can determine the charge of the peptide ion. The dominant peptide ions in proteomic studies are those with charges 2 and 3. For peptide ions with charge 2, 69\% of the ions dissociate between 10 and $90 \%, 17 \%$ dissociate $<10 \%$, and $14 \%$ dissociate $>90 \%$. Thus, a majority of these ions give a reasonable extent of dissociation at the voltage predicted from their $\mathrm{m} / \mathrm{z}$ values. Ions with charge 3, as expected, dissociate more readily and thus only $8 \%$ of them have dissociation $<10 \%$ but $44 \%$ dissociate $>90 \%$; and ions with charge 1 show the reverse trend: $73 \%$ dissociate $<10 \%$ and only $1.5 \%$ dissociate $>90 \%$. These values can be improved by changing from a slope of 0.0307 for all peptides to different slopes for different charges. From our results 
we calculate an average slope of 0.042 for singly charged peptide ions, 0.032 for doubly charged, and 0.028 for triply charged ions. By using these slopes to calculate the extent of dissociation for each peptide ion at a collision voltage derived from its $\mathrm{m} / \mathrm{z}$ value and charge we find that the percentage dissociation is greatly improved for the singly charged ions, only slightly improved for the triply charged, but remained essentially the same for the doubly charged ions. This is not surprising since the doubly charged ions constitute the predominant group and have the greatest effect on the slope calculated from Figure 1. By using the slope of 0.042 for singly charged instead of the general slope 0.0307 , we find $84 \%$ of ions dissociating between 10 and $90 \%$ at the calculated collision voltage, with only $6.5 \%$ of ions dissociating $<10 \%$ and $10 \%$ of ions dissociating $>90 \%$. Clearly, one could target a higher or lower extent of average dissociation by raising or lowering the slopes above. Of course the values will need to be adjusted for different collision cell platforms: data given as supplementary information, which can be found in the electronic version of this article, can aid in this process.

\section{Conclusions}

The observation by Haller et al. [3] that extents of dissociation of doubly charged tryptic peptides depended linearly on $\mathrm{m} / \mathrm{z}$ has been confirmed and extended in this work to include other charge states and different classes of peptides. Energies for 50\% dissociation provide a straightforward measure of reactivity toward dissociation and deviations from the $V_{1 / 2}$ versus $m / z$ curve provide a convenient measure of relative reactivity for an ion of a given class. Since knowledge of the sequence is needed to apply these rules, they cannot guide "discovery" experiments where the sequence is unknown before MS/MS measurements, but they can provide estimates for dissociation of target peptides before experiment and aid in the estimation of relative collisional energies in selected reaction monitoring (SRM) experiments. More generally, the curves given here can be used to place the dissociation of any peptide ion on a relative scale and compare the reactivity of diverse ions by, in effect, separating the effect of molecular size from that of inherent reactivity.

\section{References}

1. Dongre, A. R.; Jones, J. L.; Somogyi, A.; Wysocki, V. H. Influence of Peptide Composition, Gas Phase Basicity, and Chemical Modification on Fragmentation Efficiency: Evidence for the Mobile Proton Model. J. Am. Chem. Soc. 1996, 118, 8365-8374.

2. Summerfield, S. G.; Gaskell, S. J. Fragmentation Efficiencies of Peptide Ions Following Low Energy Collisional Activation. Int. J. Mass Spectrom. 1997, 165-166, 509-521.

3. Haller, I.; Mirza, U. A.; Chait, B. T. Collision Induced Decomposition of Peptides. Choice of Collision Parameters. J. Am. Soc. Mass Spectrom. 1996, 7, 677-681.

4. Kapp, E. A.; Schutz, F.; Reid, G. E.; Eddes, J. S.; Moritz, R. L.; O’Hair, R. A. J.; Speed, T. P.; Simpson, R. J. Mining a Tandem Mass Spectrometry Database to Determine the Trends and Global Factors Influencing Peptide Fragmentation. Anal. Chem. 2003, 75, 6251-6264.

5. Huang, Y.; Triscari, J. M.; Tseng, G. C.; Pasa-Tolic, L.; Lipton, M. S.; Smith, R. D.; Wysocki, V. H. Statistical Characterization of the Charge State and Residue Dependence of Low-Energy CID Peptide Dissociation Patterns. Anal. Chem. 2005, 77, 5800-5813.

6. Kinter, M.; Sherman, N. E. Protein Sequencing and Identification Using Tandem Mass Spectrometry. Wiley-Interscience: New York, 2000; pp 162-163.

7. Thiede, B.; Lamer, S.; Mattow, J.; Siejak, F.; Dimmler, C.; Rudel, T.; Jungblut, P. R. Analysis of Missed Cleavage Sites, Tryptophan Oxidation and N-Terminal Pyroglutamylation After In-Gel Tryptic Digestion. Rapid Commun. Mass Spectrom. 2000, 14, 496-502.

8. Geoghegan, K. F.; Hoth, L. R.; Tan, D. H.; Borzillerl, K. A.; Withka, J. M.; Boyd, J. G. Cyclization of N-Terminal S-Carbamoylmethylcysteine Causing Loss of 17 Da from Peptides and Extra Peaks in Peptide Maps. I. Proteome Res. 2002, 1, 181-187.

9. Qin, J.; Chait, B. T. Preferential Fragmentation of Protonated Gas-Phase Peptide Ions Adjacent to Acidic Amino Acid Residues. J. Am. Chem. Soc. 1995, 117, 5411-5412.

10. Gu, C.; Tsaprailis, G.; Breci, L.; Wysocki, V. H. Selective Gas-Phase Cleavage at the Peptide Bond C-Terminal to Aspartic Acid in FixedCharged Derivatives of Asp-Containing Peptides. Anal. Chem. 2000, 72, 5804-5813. 